\title{
Antecipação do período de diagnose foliar em laranjeira 'Pêra' no Amazonas
}

\author{
Jairo Rafael Machado Dias( ${ }^{(1)}$, Carlos Alberto Franco Tucci(2), Paulo Guilherme Salvador Wadt( ${ }^{(3)}$, \\ Fábio Luiz Partelli( ${ }^{(4)}$, Daniel Vidal Perez ${ }^{(5)}$, Marcelo Curitiba Espindula(6) e Denis Borges Tomio(7)
}

\begin{abstract}
(1)Universidade Federal de Rondônia, Departamento de Agronomia, Avenida Norte Sul, no 7.300, Nova Morada, CEP 76940-000 Rolim de Moura, RO. E-mail: jairorafaelmdias@hotmail.com ${ }^{(2)}$ Universidade Federal do Amazonas, Programa de Pós-graduação em Agronomia Tropical, Avenida Gal. Rodrigo O. J. Ramos, no 3.000, Coroado I, CEP 69077-000 Manaus, AM. E-mail: ctucci@ufam.edu.br ${ }^{(3)}$ Embrapa Acre, Rodovia BR 364, Caixa Postal 321, Km 14, CEP 69900-056 Rio Branco, AC. E-mail: paulogswadt@dris.com.br (4)Universidade Federal do Espírito Santo, Rodovia BR 101 Norte, Km 60, Bairro Litorâneo, CEP 29932-540 São Mateus, ES. E-mail: partelli@yahoo.com.br ${ }^{(5)}$ Embrapa Solos, Rua Jardim Botânico, no1.024, CEP 22426-000 Rio de Janeiro, RJ. E-mail: daniel.perez@embrapa.br ${ }^{(6)}$ Embrapa Rondônia, BR 364, Km 5,5, Zona Rural, CEP 76815-800 Porto Velho, RO. E-mail: marcelo.espindula@embrapa.br (7)Universidade Federal do Acre, Programa de Pós-graduação em Produção Vegetal, Caixa Postal 500, CEP 69915-900 Rio Branco, AC. E-mail: denis.tomio@gmail.com
\end{abstract}

Resumo - O objetivo deste trabalho foi avaliar a possibilidade de se antecipar o período de realização da diagnose foliar em laranjeira 'Pêra'. Vinte e sete pomares representativos da região produtora de laranja do Estado do Amazonas foram monitorados durante o ano agrícola de 2011/2012. Foram realizadas diagnoses da composição nutricional (CND) em amostras foliares retiradas durante a floração e quando a árvore apresentava frutos com três e seis meses de idade (época tradicional para o monitoramento nutricional). Pomares com produtividade superior a $25 \mathrm{Mg} \mathrm{ha}^{-1}$ foram selecionados para o estabelecimento dos padrões de referência. O estado nutricional da laranja variou com o estádio fenológico no qual se realizou a amostragem foliar, o que fez com que fosse necessário estabelecer normas CND para cada período. Com a antecipação da diagnose para o período de floração, observouse aumento nas concentrações foliares de N, P, $\mathrm{K}$ e Cu diminuição e nas de $\mathrm{Ca}$. A antecipação da diagnose foliar em laranja 'Pêra' depende da geração de padrões nutricionais CND específicos para cada época de amostragem.

Termos para indexação: Citrus limonia, Citrus sinensis, amostragem foliar, DRIS, índice de balanço nutricional.

\section{Anticipation of the period for foliar diagnosis in 'Pêra' orange tree in Amazon state, Brazil}

\begin{abstract}
The objective of this work was to evaluate the possibility of anticipating the period for foliar diagnosis in 'Pêra' orange tree. Twenty seven representative orchards from the orange production region of the state of Amazonas, Brazil, were monitored during the crop season of 2011/2012. Nutritional composition diagnoses (CND) were performed in leaf samples taken in the flowering stage and when the tree had fruits with three and six months of age (traditional period for nutritional monitoring). Orchards with productivity higher than $25 \mathrm{Mg} \mathrm{ha}^{-1}$ were selected for establishing reference standards. The nutritional status of orange varied with the foliar sample period, which made it necessary to establish specific CND norms for each period. With the anticipation of foliar diagnosis to the flowering period, it was observed that leaf concentrations of $\mathrm{N}, \mathrm{P}, \mathrm{K}$, and $\mathrm{Cu}$ increased and the ones of $\mathrm{Ca}$ decreased. The anticipation of 'Pêra' orange foliar diagnosis depends on CND nutritional standards specific to each sampling period.
\end{abstract}

Index terms: Citrus limonia, Citrus sinensis, leaf sampling, DRIS, nutritional balance index.

\section{Introdução}

A citricultura tem apresentado elevados índices de crescimento na Amazônia brasileira durante a última década, tendo alcançado 18 mil hectares e produção bruta de 265 mil toneladas de frutos ao ano. No Estado do Amazonas, segundo maior produtor da região Norte, cultiva-se tradicionalmente a laranja 'Pêra' (Instituto Brasileiro de Geografia e Estatística, 2011).
Essa variedade tem sido preferida pelos produtores por apresentar boa adaptação às condições edafoclimáticas regionais.

A ampliação na área cultivada, no entanto, não tem sido acompanhada pela geração de informações locais quanto à demanda de nutrientes pelas plantas, que viabilizariam a avaliação técnica do estado nutricional da frutífera na região. A ausência dessas informações faz com que a recomendação de adubação para a cultura, na 
Amazônia brasileira, seja bastante empírica (Fernandes et al., 2010). Dessa forma, o monitoramento nutricional das plantas a partir da análise foliar da laranjeira, apesar de ser uma técnica de simples adoção, não conta com critérios de interpretação específicos para a região amazônica e depende de valores publicados na literatura, em condições edafoclimáticas distintas.

O sistema integrado de diagnose e recomendação (DRIS) constitui uma alternativa adequada para esses casos, uma vez que permite a obtenção direta de padrões nutricionais, a partir do monitoramento dos teores foliares de pomares comerciais (Bataglia, 1989; Santana et al., 2008). A não exigência de experimentação diminui significativamente o custo e o tempo necessário para a obtenção desses padrões e tem motivado, cada vez mais, pesquisas com DRIS, como, por exemplo, para cafeeiros (Wadt \& Dias, 2012) e cupuaçuzeiros (Dias et al., 2010, 2011), em Rondônia, ou para mangueiras (Wadt \& Silva, 2010), na região do Semiárido nordestino.

O método DRIS varia, quanto às relações nutricionais utilizadas, que podem ser bivariadas ou multivariadas. O DRIS com uso de relações multivariadas foi proposto como um sistema de diagnose distinto, denominado de diagnose da composição nutricional (CND). O CND corresponde ao logaritmo natural da relação entre o nutriente avaliado e a média geométrica da composição nutricional na amostra foliar (Parent, 2011). Portanto, o CND, diferentemente do método DRIS original (Beaufils, 1973), não considera a relação entre os teores de dois nutrientes quaisquer.

$\mathrm{O}$ método CND vem sendo utilizado em diversas culturas comerciais, em diferentes regiões do Brasil, como: arroz irrigado no Rio Grande do Sul (Wadt et al., 2013), cafeeiros em Rondônia (Wadt \& Dias, 2012), eucalipto em Minas Gerais (Silva et al., 2005), mangueiras no Semiárido nordestino (Politi et al., 2013), laranjeiras no Amazonas (Dias et al., 2013) e no Estado de São Paulo (Camacho et al., 2012).

Um fator determinante para a eficiência do DRIS na avaliação nutricional de cultivos comerciais referese ao critério utilizado para a obtenção das normas, que equivalem aos padrões de referência para as relações bivariadas ou multivariadas. Essas normas devem ser obtidas de plantas sadias, com histórico de boa produtividade e, frequentemente, para condições ecofisiológicas ou de manejo específicas (Creste \& Nakagawa, 1997; Mourão Filho et al., 2002; Partelli et al., 2006). Na região amazônica, a carência de informações nutricionais para laranjeiras faz com que os valores de referência disponíveis para São Paulo (Quaggio et al., 2005) sejam utilizados. O uso desses valores pode diminuir a eficácia dos diagnósticos, porque foram estabelecidos em condições de cultivo distintas da Amazônia, conforme já constatado para esta fruteira (Santana et al., 2007; Fernandes et al., 2010).

Além disso, esses padrões foram obtidos em pomares com frutos de seis meses de idade e devem ser utilizados na análise de amostras retiradas nessas condições. Contudo, a amostragem nessa época torna o manejo das adubações limitado, uma vez que, nessa idade, as principais fertilizações já foram realizadas.

O objetivo deste trabalho foi avaliar a possibilidade de se antecipar o período de realização da diagnose foliar em laranjeira 'Pêra'.

\section{Material e Métodos}

Foram monitorados 27 pomares comerciais de laranja 'Pêra' (Citrus sinensis L. Osbeck), enxertados em limoeiro 'Cravo' (C. limonia Osbeck), representativos da região produtora desta fruta no Estado do Amazonas, nos limites do Município de Rio Preto da Eva $\left(02^{\circ} 41^{\prime} 56^{\prime \prime} \mathrm{S}, 59^{\circ} 42^{\prime} 00^{\prime \prime} \mathrm{W}\right)$, durante o ano agrícola de 2011/2012. Os pomares apresentavam de 236 a 357 plantas por hectare, com idade entre 5 e 15 anos. O clima da região, segundo a classificação de Köppen é do tipo Af, tropical úmido, com temperatura média anual de $26^{\circ} \mathrm{C}$ e precipitação pluvial média de $2.550 \mathrm{~mm}$ por ano. O período chuvoso corresponde aos meses de dezembro a abril, com acúmulo de chuvas no primeiro trimestre do ano. $\mathrm{O}$ período mais quente compreende os meses de agosto a outubro.

Em cada pomar monitorado, foram realizadas amostragens foliares em três épocas. A primeira foi realizada por ocasião do florescimento principal (setembro de 2011), e as duas seguintes foram feitas quando as plantas apresentavam frutos com três e seis meses de idade, em dezembro de 2011 e março de 2012, respectivamente.

Foram retiradas amostras compostas de 25 árvores, num total amostrado de 100 folhas recém-amadurecidas, coletadas na terceira posição de lançamento, a partir do ápice de ramos floríferos. As amostras foram retiradas nas faces da copa correspondentes aos quatro pontos 
cardeais, sempre na altura mediana da planta, conforme recomendação de Quaggio et al. (2005). O material vegetal coletado foi acondicionado em sacos de papel e transportado para o laboratório, onde foi lavado, seco, moído e submetido à análise quanto aos teores totais de N, P, K, Ca, Mg, B, Cu, Fe, Mn e Zn (Carmo et al., 2000).

Os pomares com produtividade de frutos na safra principal superior a $25 \mathrm{Mg} \mathrm{ha}^{-1}$ (junho de 2012) foram considerados como de alta produtividade e utilizados para o estabelecimento dos padrões nutricionais. O rendimento de $25 \mathrm{Mg}$ ha $^{-1}$ foi utilizado como critério de corte, por superar, em mais de $50 \%$, a produtividade média da fruteira no Amazonas (Instituto Brasileiro de Geografia e Estatística, 2011).

As características do solo, na camada de $0-20 \mathrm{~cm}$, nos pomares de alta produtividade, apresentaram os seguintes valores médios: $\mathrm{pH}$ em $\mathrm{CaCl}_{2}$ de 5,7; $\mathrm{P}$ (Melich-1) de 5,6 mg dm${ }^{-3}$; $\mathrm{Ca}, \mathrm{Mg}, \mathrm{K}$ e acidez potencial, respectivamente, de 2,3, 1,2, 0,2 e 2,9 $\mathrm{cmol}_{\mathrm{c}} \mathrm{dm}^{-3}$; carbono orgânico oxidável de 3,0 dag $\mathrm{kg}^{-1}$; e teor de argila de $593 \mathrm{dag}^{\mathrm{kg}}{ }^{-1}$. Nos pomares de baixa produtividade, os resultados da análise foram: $\mathrm{pH}$ em $\mathrm{CaCl}_{2}$ de 5,4; $\mathrm{P}$ de $1,2 \mathrm{mg} \mathrm{dm}^{-3} ; \mathrm{Ca}, \mathrm{Mg}, \mathrm{K}$ e acidez potencial, respectivamente, de 1,7, 1,1, 0,2 e 4,5 $\mathrm{cmol}_{\mathrm{c}} \mathrm{dm}^{-3}$; carbono orgânico oxidável de 3,2 dag $\mathrm{kg}^{-1}$; e teor de argila de $492 \mathrm{dag} \mathrm{kg}^{-1}$. As análises foram realizadas conforme Donagema (2011).

A concentração foliar dos nutrientes, em todos os pomares, independentemente da época de amostragem, foi ajustada para uma mesma unidade de medida (dag $\mathrm{kg}^{-1}$ ). Em seguida, calculou-se o valor do complemento dos nutrientes para o total da biomassa foliar (valor $\mathrm{R}$ ), conforme a expressão: $\mathrm{R}=100-(\mathrm{vN}+\mathrm{vP}+\mathrm{vK}+\mathrm{vCa}+\mathrm{vMg}+\mathrm{vB}+$ $\mathrm{vCu}+\mathrm{vFe}+\mathrm{vMn}+\mathrm{vZn}$ ), em que $\mathrm{R}$ é o valor do complemento para 100 dag $\mathrm{kg}^{-1}$ de matéria seca em relação à soma dos teores dos nutrientes $\mathrm{v}_{\mathrm{i}}$ ( $\mathrm{i}=\mathrm{N}, \ldots, \mathrm{Zn}$ ), em dag $\mathrm{kg}^{-1}$; e vN, vP, vK, vCa, vMg, $\mathrm{vB}, \mathrm{vCu}, \mathrm{vFe}, \mathrm{vMn}$ e $\mathrm{vZn}$ representam os teores de $\mathrm{N}$, $\mathrm{P}, \mathrm{K}, \mathrm{Ca}, \mathrm{Mg}, \mathrm{B}, \mathrm{Cu}, \mathrm{Fe}, \mathrm{Mn}$ e $\mathrm{Zn}$, respectivamente.

De posse da média geométrica (mGeo) calculada para os valores de cada amostra (Parent, 2011), obtevese a variável multinutriente $(\mathrm{zX})$ a partir da expressão: $\mathrm{zX}=\ln (\mathrm{vX} / \mathrm{mGeo})$, em que $\mathrm{zX}$ representa o valor da relação multivariada de cada um dos nutrientes avaliados (vX). Com os valores de $\mathrm{zX}$ em cada pomar de alta produtividade, nas três épocas de amostragem foliar, calcularam-se os parâmetros descritivos - média aritmética (mX) e desvio-padrão $(\mathrm{sX})$ - e as normas CND para cada estádio fenológico da laranjeira 'Pêra'.

Obtida as normas, os índices CND foram calculados pela relação multivariada log-centrada (Parent, 2011): I_X $=(\mathrm{zX}-\mathrm{mX}) / \mathrm{sX}$, em que I_X representa o índice CND; mX é a norma média; e sX é a norma do desvio-padrão, para cada um dos nutrientes avaliados.

O somatório, em módulo, dos índices CND dos nutrientes, em cada pomar de baixa produtividade e em cada estádio fenológico, constituiu o índice de balanço nutricional (IBN) dos pomares. $\mathrm{O}$ índice de balanço nutricional médio (IBNm) foi obtido dividindo-se o valor do IBN pelo número de nutrientes avaliados. $\mathrm{O}$ nutriente foi considerado nutricionalmente equilibrado quando o IBN foi menor que o IBNm; insuficiente, quando o IBN foi maior que o IBNm e o índice CND menor que zero; e excessivo, ou na fase de consumo de luxo, quando o IBN foi maior que o IBNm e o índice CND maior que zero (Wadt, 2005).

Os cálculos das normas CND, IBN, IBNm foram realizados em planilha eletrônica. As estatísticas descritivas e o teste de Duncan, a 5\% de probabilidade, foram obtidos com o programa estatístico Assistat, versão 6.2 (UFCG, Campina Grande, Brasil). Os valores das normas CND para cada nutriente, no florescimento pleno e aos três e seis meses da frutificação, foram comparados pelo teste $\mathrm{t}$ de duas amostras, tendo-se considerado o grau de liberdade de 32 ( $2 \mathrm{~N}-2$, em que $\mathrm{N}$ é o tamanho da amostra) e o valor de $\mathrm{t}$ tabelado, de 1,3086.

Nos pomares de baixa produtividade, os diagnósticos nutricionais para os três estádios fenológicos foram confrontados entre si, tendo-se computado os casos de concordância, para cada amostra foliar e nutriente. Foram considerados diagnósticos concordantes quando as diferentes épocas de amostragem foliar resultaram no mesmo diagnóstico (deficiência, equilíbrio ou excesso). Os resultados foram expressos em percentagem de concordância entre os diagnósticos.

\section{Resultados e Discussão}

Dos 27 pomares comerciais de laranja 'Pêra' monitorados, 17 apresentaram produtividade superior a $25 \mathrm{Mg} \mathrm{ha}^{-1}$ na safra principal (junho de 2012) e foram considerados de alta produtividade para a região de estudo, tendo sido utilizados para o estabelecimento 
dos padrões foliares (Tabela 1). Os dez pomares restantes apresentaram baixa produtividade e foram utilizados para realização dos diagnósticos. A base de dados utilizada pode ser considerada pequena para obtenção das normas CND, mas as informações nutricionais foram obtidas de pomares que representam adequadamente os sistemas de cultivo do Amazonas. Walworth et al. (1988) já haviam demonstrado que normas desenvolvidas com apenas dez observações, desde que representativas, são suficientes para proporcionar diagnósticos nutricionais semelhantes aos obtidos com o uso de base de dados ampla.

Nos pomares de alta produtividade, os teores foliares médios de N, P, B e Fe foram considerados adequados; os de $\mathrm{K}, \mathrm{Ca}, \mathrm{Mn}$ e $\mathrm{Zn}$, baixos; e os de $\mathrm{Mg}$ e $\mathrm{Cu}$, excessivos, para laranjeiras com frutos de seis meses de idade (Quaggio et al., 2005). As concentrações foliares de $\mathrm{N}, \mathrm{P}, \mathrm{K} \mathrm{e} \mathrm{Cu}$ foram maiores na fase do florescimento do que nos demais períodos amostrados, enquanto os teores de Mg mantiveram-se relativamente estáveis, e os de Ca, Mn e Zn aumentaram na fase de frutificação (Tabela 1).

Mattos Junior et al. (2003) também observaram diminuição nas concentrações foliares de N, P e K à medida que o florescimento da laranjeira 'Hamlin' avançava. Esses resultados podem ser explicados pelo fato de N, K e P serem, nesta ordem, os elementos mais exportados nos frutos da laranjeira (Boaretto et al., 2007). Assim, como os órgãos reprodutivos são drenos preferenciais, a tendência é que estes elementos sejam mobilizados da folha para os frutos. Já o $\mathrm{Ca}$, por ter restrita mobilidade no floema, acumula-se no tecido foliar com o desenvolvimento da planta.

A variação nutricional nas folhas, conforme $\mathrm{o}$ estádio fenológico no qual foi realizada a amostragem, pode estar associada a diversos fatores relacionados à fisiologia da planta, como exigências provenientes da frutificação, da emissão de ramos vegetativos e reprodutivos. Também pode estar relacionada a variáveis meteorológicas, como temperatura, radiação, precipitação, entre outros, uma vez que, tais variáveis, podem interferir no comportamento fisiológico e nutricional das plantas (Fageria et al., 2009).

Assim como os teores foliares, normas nutricionais CND também variaram conforme o estádio fenológico no qual foi realizada a amostragem (Tabela 2). Entre o pleno florescimento e os frutos com três meses de idade, apenas as CND quanto a P, K, Ca e Zn diferiram. As normas para $\mathrm{N}$ também diferiram significativamente quando analisaram-se os períodos do florescimento e dos frutos com seis meses. Entre as duas épocas de frutificação, foram observadas diferenças para K, $\mathrm{B}$ e Fe. Distinções entre normas DRIS afetadas pelo período de coleta do material vegetal foram relatadas para cafeeiro arábica (Partelli et al., 2007).

Recentemente, diversos pesquisadores têm estabelecido padrões foliares por meio do DRIS, para diversas culturas, como cafeeiros (Partelli et al., 2006; Wadt \& Dias, 2012), cana-de-açúcar (McCray et al., 2010) e laranjeiras (Santana et al., 2008; Camacho et al., 2012); entretanto, nesses estudos, têm-se realizado as amostragens nas épocas sugeridas pela literatura, conforme o estádio fenológico da planta. No Brasil, o diagnóstico nutricional da laranjeira é feito com valores de referência para plantas com frutos de seis meses de idade. Essa época geralmente coincide com os meses de fevereiro a março, em diversas regiões brasileiras (Santana et al., 2007; Fernandes et al., 2010; Auler et al., 2011). Portanto, esses valores podem ser inadequados para o manejo das adubações, uma vez que estas são indicadas para o início da floração, entre setembro e outubro (Almeida \& Baumgartner, 2002). Dessa forma, com padrões foliares obtidos em plantas com frutos de seis meses de idade, eventuais problemas nutricionais serão diagnosticados tardiamente.

Tabela 1. Teores foliares de laranjeira 'Pêra' nos pomares de alta produtividade $\left(>25 \mathrm{Mg} \mathrm{ha}^{-1}\right)$, em amostradas retiradas de plantas nos estádios de florescimento pleno e, com frutos de três e seis meses de idade ${ }^{(1)}$.

\begin{tabular}{|c|c|c|c|c|c|c|c|c|c|c|}
\hline \multirow[t]{2}{*}{ Época } & $\mathrm{N}$ & $\mathrm{P}$ & $\mathrm{K}$ & $\mathrm{Ca}$ & $\mathrm{Mg}$ & $\mathrm{B}$ & $\mathrm{Cu}$ & $\mathrm{Fe}$ & $\mathrm{Mn}$ & $\mathrm{Zn}$ \\
\hline & \multicolumn{5}{|c|}{ 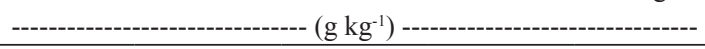 } & \multicolumn{5}{|c|}{ 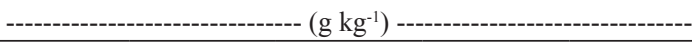 } \\
\hline Florescimento & $30,62 \mathrm{a}$ & $1,42 \mathrm{a}$ & $12,15 \mathrm{a}$ & $17,59 \mathrm{c}$ & $2,97 b$ & $63,68 b$ & $25,13 a$ & $68,27 \mathrm{a}$ & $11,15 b$ & $13,53 b$ \\
\hline Frutos com três meses & $26,88 b$ & $1,21 \mathrm{~b}$ & $10,15 b$ & $19,72 b$ & $3,33 a$ & $58,12 \mathrm{c}$ & $17,55 \mathrm{~b}$ & $56,13 b$ & $3,52 \mathrm{ab}$ & $19,36 \mathrm{ab}$ \\
\hline Frutos com seis meses & $26,02 b$ & $1,27 \mathrm{~b}$ & $6,40 \mathrm{c}$ & $22,27 \mathrm{a}$ & $3,09 \mathrm{ab}$ & $70,33 \mathrm{a}$ & $19,08 \mathrm{~b}$ & $70,36 \mathrm{a}$ & $16,21 \mathrm{a}$ & $23,73 \mathrm{a}$ \\
\hline $\mathrm{CV}(\%)$ & 18,84 & 8,50 & 14,68 & 7,95 & 14,10 & 10,31 & 41,18 & 23,02 & 43,62 & 58,92 \\
\hline
\end{tabular}

${ }^{(1)}$ Médias seguidas de letras iguais não diferem pelo teste de Duncan, a $5 \%$ de probabilidade. 
A disponibilidade de valores de referência nutricional para estádios fenológicos precoces, pode antecipar o diagnóstico nutricional e atender ao manejo da adubação antes que problemas nutricionais venham a ocorrer.

As diagnoses nutricionais realizadas com os valores obtidos nas diferentes épocas diferiram para um mesmo pomar (Tabela 3). Esse resultado, associado às diferenças observadas nas normas $\mathrm{CND}$, reforça a importância da disponibilização de padrões de referência para períodos específicos da fenologia das plantas. Os índices CND negativos e positivos indicam, respectivamente, deficiência e excesso nutricional, à medida que se distanciam do valor zero, e são úteis para identificar os nutrientes mais limitantes, seja por deficiência (menor índice CND) ou por excesso (maior índice CND) (Mourão Filho, 2004).

O IBN nos pomares de baixa produtividade variou entre 4 e 26, nos diferentes estádios fenológicos avaliados. Mourão Filho \& Azevedo (2003) observaram alta correlação $\left(\mathrm{R}^{2}=0,83\right)$ entre a produtividade e o IBN em laranjeira 'Valência' enxertada sob 'Trifoliata' (Poncirus trifoliata). Isso indica que o desequilíbrio nutricional (altos valores de IBN) está associado a baixas produtividades.

O grau de concordância dos diagnósticos realizados nas diferentes épocas de amostragem foi inferior a $65 \%$, para a maioria dos nutrientes (Tabela 4). Isso indica que o estado nutricional dos pomares variou entre as três fases fenológicas, mesmo com o uso de normas CND específicas para cada período. As maiores variações ocorreram com $\mathrm{N}$ e $\mathrm{P}$, que tiveram graus de concordância de, no máximo, 50\% entre os diagnósticos. $\mathrm{O} \mathrm{Cu}$ foi o elemento que menos sofreu influência dos diferentes estádios fenológicos nos quais foi realizada a amostragem, com graus de concordância entre diagnósticos de 70 a 80\%. Essas variações estão relacionadas às demandas nutricionais da planta durante a frutificação ou na emissão de ramos vegetativos e reprodutivos. Os efeitos de diluição (maior acúmulo de matéria orgânica que do nutriente) e de concentração (menor acúmulo de matéria orgânica que do nutriente) são decisivos para essa variação em cada período monitorado (Jarrel \& Beverly, 1981). Resultados semelhantes foram relatados para laranjeira 'Valência' (Vasconcelos et al., 2010).

Baixos graus de concordância indicam que o estado nutricional da laranjeira 'Pêra', na região de estudo, é peculiar a cada fase fenológica e que há diferenças quanto às exigências nutricionais nas diferentes épocas do ano. A grande diferença entre os diagnósticos realizados com normas CND específicas para o estado fenológico e os realizados com normas não específicas - para uma mesma época de amostragem - confirma que as normas CND devem ser específicas para cada período de amostragem (Tabela 5). O uso de normas específicas também é recomendado para eucalipto (Silva et al., 2005) e para cafeeiros arábica (Partelli et al., 2007) e conilon (Partelli et al., 2006).

Tabela 2. Média e desvio-padrão para as normas de diagnose da composição nutricional para laranjeira 'Pêra' e contrastes entre as normas obtidas para os diferentes estádios fenológicos.

\begin{tabular}{|c|c|c|c|c|c|c|c|c|c|c|}
\hline Parâmetro & $\mathrm{N}$ & $\mathrm{P}$ & $\mathrm{K}$ & $\mathrm{Ca}$ & $\mathrm{Mg}$ & $\mathrm{B}$ & $\mathrm{Cu}$ & $\mathrm{Fe}$ & $\mathrm{Mn}$ & $\mathrm{Zn}$ \\
\hline & \multicolumn{10}{|c|}{ Florescimento pleno (FP) } \\
\hline Média & 3,58 & 0,53 & 2,65 & 3,03 & 1,23 & $-2,58$ & $-4,27$ & $-2,57$ & $-4,41$ & $-4,20$ \\
\hline \multirow[t]{2}{*}{$\underline{\text { Desvio-padrão }}$} & 0,25 & 0,16 & 0,21 & 0,29 & 0,36 & 0,24 & 1,11 & 0,34 & 0,30 & 0,29 \\
\hline & \multicolumn{10}{|c|}{ Frutos com três meses (LF3) } \\
\hline Média & 3,50 & 0,42 & 2,48 & 3,20 & 1,39 & $-2,63$ & $-4,58$ & $-2,67$ & $-4,30$ & $-3,88$ \\
\hline \multirow[t]{2}{*}{ Desvio-padrão } & 0,17 & 0,25 & 0,31 & 0,35 & 0,45 & 0,24 & 1,06 & 0,22 & 0,55 & 0,46 \\
\hline & \multicolumn{10}{|c|}{ Frutos com seis meses (LF6) } \\
\hline Média & 3,43 & 0,42 & 1,99 & 3,28 & 1,28 & $-2,48$ & $-4,57$ & $-2,49$ & $-4,09$ & $-3,79$ \\
\hline \multirow[t]{2}{*}{ Desvio-padrão } & 0,21 & 0,25 & 0,31 & 0,31 & 0,35 & 0,26 & 0,98 & 0,21 & 0,33 & 0,44 \\
\hline & \multicolumn{10}{|c|}{ Contraste } \\
\hline FP vs.LF3 & ns & * & $*$ & $*$ & ns & ns & ns & ns & ns & $*$ \\
\hline FP vs. LF6 & $*$ & $*$ & $*$ & $*$ & $\mathrm{~ns}$ & $\mathrm{~ns}$ & $\mathrm{~ns}$ & ns & $*$ & $*$ \\
\hline LF3 vs. LF6 & ns & ns & $*$ & ns & $\mathrm{ns}$ & $*$ & $\mathrm{~ns}$ & $*$ & ns & ns \\
\hline
\end{tabular}

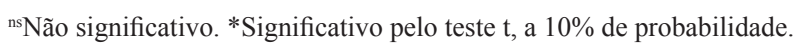


Tabela 3. Índices de diagnose da composição nutricional (índices CND) e de balanço nutricional (IBN), e diagnósticos nutricionais ${ }^{(1)}$ realizados com normas específicas para cada estádio fenológico, em pomares de laranja 'Pêra' de baixa produtividade $\left(<25 \mathrm{Mg} \mathrm{ha}^{-1}\right)$, com amostradas retiradas de plantas nos períodos do florescimento pleno e, com frutos de três e seis meses de idade.

\begin{tabular}{|c|c|c|c|c|c|c|c|c|c|c|c|}
\hline Pomar & $\mathrm{N}$ & $\mathrm{P}$ & $\mathrm{K}$ & $\mathrm{Ca}$ & $\mathrm{Mg}$ & $\mathrm{B}$ & $\mathrm{Cu}$ & $\mathrm{Fe}$ & $\mathrm{Mn}$ & $\mathrm{Zn}$ & IBN \\
\hline & \multicolumn{11}{|c|}{ Florescimento pleno } \\
\hline 1 & $0,2(0)$ & $-1,9(1)$ & $-1,1(1)$ & $-0,1(0)$ & $0,2(0)$ & $0,8(2)$ & $0,2(0)$ & $0,8(2)$ & $0,7(0)$ & $-1,3(1)$ & 7 \\
\hline 2 & $0,2(0)$ & $-1,6(1)$ & $-1,2(1)$ & $0,5(0)$ & $0,9(2)$ & $1,2(2)$ & $-0,1(0)$ & $0,0(0)$ & $0,3(0)$ & $-1,3(1)$ & 7 \\
\hline 3 & $-0,8(1)$ & $-1,2(1)$ & $-0,4(0)$ & $0,2(0)$ & $0,4(0)$ & $2,0(2)$ & $0,0(0)$ & $0,4(0)$ & $0,5(0)$ & $-1,8(1)$ & 8 \\
\hline 4 & $0,1(0)$ & $-1,0(1)$ & $0,5(0)$ & $0,1(0)$ & $0,7(2)$ & $1,4(2)$ & $-0,2(0)$ & $0,2(0)$ & $-0,2(0)$ & $-1,5(1)$ & 6 \\
\hline 5 & $0,3(0)$ & $1,3(2)$ & $0,6(0)$ & $-0,3(0)$ & $0,8(2)$ & $-1,3(1)$ & $0,1(0)$ & $-1,1(1)$ & $-0,2(0)$ & $0,0(0)$ & 6 \\
\hline 6 & $0,2(0)$ & $0,7(2)$ & $-0,3(0)$ & $-0,3(0)$ & $1,0(2)$ & $-0,9(1)$ & $0,3(0)$ & $-0,5(0)$ & $-0,5(1)$ & $-0,5(0)$ & 5 \\
\hline 7 & $1,3(2)$ & $2,3(2)$ & $1,3(2)$ & $-0,6(0)$ & $0,9(0)$ & $-0,5(0)$ & $-0,3(0)$ & $-0,7(0)$ & $-1,2(1)$ & $-0,5(0)$ & 10 \\
\hline 8 & $-2,1(0)$ & $-3,5(1)$ & $-3,4(1)$ & $-1,8(0)$ & $-2,0(0)$ & $-1,8(0)$ & $1,3(0)$ & $-0,5(0)$ & $4,7(2)$ & $5,1(2)$ & 26 \\
\hline 9 & $-2,0(0)$ & $-3,1(1)$ & $-2,5(0)$ & $-2,0(0)$ & $-1,8(0)$ & $-1,9(0)$ & $1,3(0)$ & $-1,5(0)$ & $4,4(2)$ & $5,2(2)$ & 26 \\
\hline \multirow[t]{2}{*}{10} & $-3,1(1)$ & $-3,2(1)$ & $-1,8(0)$ & $-2,0(0)$ & $-1,4(0)$ & $-1,8(0)$ & $1,2(0)$ & $-1,2(0)$ & $4,3(2)$ & $4,8(2)$ & 25 \\
\hline & \multicolumn{11}{|c|}{ Frutos com três meses } \\
\hline 1 & $1,5(2)$ & $-0,2(0)$ & $-0,3(0)$ & $0,5(0)$ & $0,7(2)$ & $0,2(0)$ & $-0,5(0)$ & $1,1(2)$ & $0,1(0)$ & $-1,2(1)$ & 6 \\
\hline 2 & $2,0(2)$ & $0,1(0)$ & $0,1(0)$ & $-0,1(0)$ & $0,4(2)$ & $0,1(0)$ & $-0,2(0)$ & $0,1(0)$ & $-0,2(0)$ & $-0,8(1)$ & 4 \\
\hline 3 & $0,4(0)$ & $0,4(0)$ & $1,0(2)$ & $-0,2(0)$ & $0,4(0)$ & $0,6(2)$ & $-0,4(0)$ & $0,2(0)$ & $-0,2(0)$ & $-0,9(1)$ & 5 \\
\hline 4 & $-0,2(0)$ & $-0,1(0)$ & $0,9(2)$ & $-0,6(1)$ & $0,1(0)$ & $1,1(2)$ & $-0,2(0)$ & $1,2(2)$ & $-0,3(0)$ & $-0,8(1)$ & 5 \\
\hline 5 & $1,0(0)$ & $1,1(0)$ & $1,9(2)$ & $-1,2(1)$ & $0,3(0)$ & $-1,7(1)$ & $0,7(0)$ & $-1,2(1)$ & $-0,9(0)$ & $-0,8(0)$ & 11 \\
\hline 6 & $1,4(2)$ & $0,8(0)$ & $1,1(2)$ & $-1,0(1)$ & $0,3(0)$ & $-1,2(1)$ & $0,8(0)$ & $-0,6(0)$ & $-0,8(0)$ & $-1,1(1)$ & 9 \\
\hline 7 & $-1,4(1)$ & $-0,3(0)$ & $0,6(0)$ & $-0,7(1)$ & $0,0(0)$ & $-0,6(0)$ & $1,3(2)$ & $-0,5(0)$ & $-0,2(0)$ & $-1,1(1)$ & 7 \\
\hline 8 & $0,1(0)$ & $0,4(0)$ & $0,4(2)$ & $-0,7(1)$ & $-0,6(1)$ & $-0,6(1)$ & $0,4(2)$ & $-0,4(1)$ & $0,1(0)$ & $0,4(0)$ & 4 \\
\hline 9 & $-2,3(1)$ & $-1,7(1)$ & $-1,0(0)$ & $-1,5(0)$ & $-1,7(0)$ & $-1,1(0)$ & $1,3(0)$ & $-1,9(1)$ & $2,0(2)$ & $2,6(2)$ & 17 \\
\hline \multirow[t]{2}{*}{10} & $-1,1(1)$ & $-0,6(0)$ & $-0,6(0)$ & $-0,3(0)$ & $0,2(0)$ & $-1,1(1)$ & $1,2(2)$ & $1,1(2)$ & $-0,2(0)$ & $-0,9(1)$ & 7 \\
\hline & \multicolumn{11}{|c|}{ Frutos com seis meses } \\
\hline 1 & $-2,7(1)$ & $-2,1(1)$ & $-1,1(0)$ & $-1,0(0)$ & $-1,0(0)$ & $-0,5(0)$ & $1,5(0)$ & $-2,2(1)$ & $1,9(2)$ & $2,4(2)$ & 16 \\
\hline 2 & $1,7(2)$ & $0,2(0)$ & $0,9(2)$ & $-0,5(0)$ & $0,5(0)$ & $-1,0(1)$ & $0,1(0)$ & $0,8(2)$ & $-1,1(1)$ & $-0,7(1)$ & 7 \\
\hline 3 & $1,0(2)$ & $0,4(0)$ & $1,3(2)$ & $-0,4(0)$ & $0,5(0)$ & $0,3(0)$ & $-0,4(0)$ & $0,2(0)$ & $-0,7(1)$ & $-0,7(1)$ & 6 \\
\hline 4 & $1,0(2)$ & $0,7(2)$ & $1,9(2)$ & $-1,1(1)$ & $0,6(0)$ & $0,1(0)$ & $-0,1(0)$ & $0,2(0)$ & $-1,1(1)$ & $-0,9(1)$ & 7 \\
\hline 5 & $0,4(0)$ & $0,7(0)$ & $1,6(2)$ & $-0,8(0)$ & $0,8(0)$ & $-1,3(1)$ & $0,5(0)$ & $-0,2(0)$ & $-1,3(1)$ & $-0,8(1)$ & 8 \\
\hline 6 & $0,1(0)$ & $0,3(0)$ & $1,3(2)$ & $-0,5(0)$ & $1,1(0)$ & $-1,7(1)$ & $0,8(0)$ & $0,6(0)$ & $-1,3(1)$ & $-1,5(1)$ & 9 \\
\hline 7 & $0,0(0)$ & $0,8(0)$ & $2,5(2)$ & $-1,1(1)$ & $0,7(0)$ & $-1,6(1)$ & $0,6(0)$ & $-0,1(0)$ & $-1,4(1)$ & $-1,4(1)$ & 10 \\
\hline 8 & $-1,0(1)$ & $-0,9(1)$ & $0,1(0)$ & $-0,6(0)$ & $-0,6(0)$ & $0,7(0)$ & $0,8(2)$ & $-1,8(1)$ & $0,8(2)$ & $0,6(0)$ & 8 \\
\hline 9 & $-0,4(0)$ & $-0,8(0)$ & $0,2(0)$ & $-1,2(1)$ & $-1,1(0)$ & $-0,2(0)$ & $1,0(2)$ & $-2,4(1)$ & $0,8(0)$ & $1,1(2)$ & 9 \\
\hline 10 & $-1,7(1)$ & $-2,1(1)$ & $-1,3(0)$ & $-1,4(1)$ & $-1,5(0)$ & $-0,7(0)$ & $2,0(2)$ & $-0,4(0)$ & $1,0(0)$ & $1,5(2)$ & 13 \\
\hline
\end{tabular}

${ }^{(1)}$ Valores entre parênteses representam os diagnósticos: 0, equilíbrio; 1, deficiência; e 2, excesso nutricional.

Tabela 4. Grau de concordância entre os diagnósticos realizados com folhas amostradas nos períodos do florescimento pleno (FP) e de frutos com três (LF3) e seis meses de idade (LF6), com uso de normas de diagnose da composição nutricional específicas para cada estádio fenológico da laranjeira 'Pêra'.

\begin{tabular}{lccc}
\hline Nutriente & FP vs. LF3 & FP vs. LF6 & LF3 vs. LF6 \\
\hline $\mathrm{N}$ & 40 & 40 & 30 \\
$\mathrm{P}$ & 10 & 30 & 50 \\
$\mathrm{~K}$ & 20 & 30 & 70 \\
$\mathrm{Ca}$ & 50 & 60 & 50 \\
$\mathrm{Mg}$ & 50 & 60 & 70 \\
$\mathrm{~B}$ & 60 & 50 & 40 \\
$\mathrm{Cu}$ & 70 & 70 & 80 \\
$\mathrm{Fe}$ & 60 & 50 & 50 \\
$\mathrm{Mn}$ & 60 & 30 & 10 \\
$\mathrm{Zn}$ & 60 & 50 & 70 \\
\hline
\end{tabular}


Tabela 5. Grau de concordância entre os diagnósticos realizados com normas de diagnose da composição nutricional específicas e não específicas para laranjeira 'Pêra', nos estádios do florescimento pleno (FP) e de frutos com três (LF3) e seis meses de idade (LF6).

\begin{tabular}{|c|c|c|c|c|c|c|c|c|c|c|c|}
\hline Diagnóstico $^{(1)}$ & $\mathrm{N}$ & $\mathrm{P}$ & $\mathrm{K}$ & $\mathrm{Ca}$ & $\mathrm{Mg}$ & B & $\mathrm{Cu}$ & $\mathrm{Fe}$ & $\mathrm{Mn}$ & $\mathrm{Zn}$ & Média \\
\hline & \multicolumn{11}{|c|}{ Florescimento pleno } \\
\hline FP vs. LF3 & 50 & 50 & 50 & 50 & 50 & 100 & 100 & 80 & 80 & 80 & 69 \\
\hline \multirow[t]{2}{*}{ FP vs. LF6 } & 60 & 20 & 30 & 50 & 60 & 70 & 100 & 80 & 90 & 90 & 65 \\
\hline & \multicolumn{11}{|c|}{ Frutos com três meses de idade } \\
\hline LF3 vs. FP & 70 & 50 & 50 & 30 & 60 & 90 & 70 & 60 & 70 & 20 & 57 \\
\hline \multirow[t]{2}{*}{ LF3 vs. LF6 } & 70 & 90 & 60 & 70 & 70 & 70 & 90 & 60 & 40 & 90 & 71 \\
\hline & \multicolumn{11}{|c|}{ Frutos com seis meses idade } \\
\hline LF6 vs. FP & 70 & 80 & 10 & 60 & 40 & 80 & 50 & 70 & 40 & 50 & 55 \\
\hline LF6 vs. LF3 & 100 & 70 & 10 & 80 & 90 & 60 & 70 & 50 & 20 & 90 & 64 \\
\hline
\end{tabular}

${ }^{(1)}$ Neste caso, as primeiras siglas representam os diagnósticos realizados com normas específicas

\section{Conclusões}

1. O estado nutricional da laranjeira 'Pêra' cultivada no Amazonas varia conforme a época de amostragem foliar, utilizando-se a diagnose da composição nutricional (CND).

2. Durante o florescimento pleno, as concentrações foliares de N, P, K e Cu são maiores e as de Ca menores que nos demais períodos fenológicos avaliados.

3. A antecipação da diagnose foliar em laranja 'Pêra' depende de padrões nutricionais CND específicos para cada estádio fenológico.

\section{Referências}

ALMEIDA, M.C. de; BAUMGARTNER, J.G. Efeitos da adubação nitrogenada e potássica na produção e na qualidade de frutos de laranjeira-'Valência'. Revista Brasileira de Fruticultura, v.24, p.282-284, 2002. DOI: 10.1590/S0100-29452002000100063.

AULER, P.A.M.; NEVES, C.S.V.J.; FIDALSKI, J.; PAVAN, M.A. Calagem e desenvolvimento radicular, nutrição e produção de laranja 'Valência' sobre porta-enxertos e sistemas de preparo do solo. Pesquisa Agropecuária Brasileira, v.46, p.254-161, 2011. DOI: 10.1590/S0100-204X2011000300005.

BATAGLIA, O.C. DRIS-Citros: uma alternativa para avaliar a nutrição das plantas. Laranja, v.10, p.565-576, 1989.

BEAUFILS, E.R. Diagnosis and recommendation integrated system (DRIS). Bloemfontein: University of Natal, 1973. 132p.

BOARETTO, R.M.; MATTOS JUNIOR, D.; TRIVELIN, P.C.O.; MURAOKA, T.; BOARETTO, A.E. Acúmulo de nutrientes e destino do nitrogênio $(15 \mathrm{~N})$ aplicado em pomar jovem de laranjeira. Revista Brasileira de Fruticultura, v.29, p.600-605, 2007. DOI: 10.1590/S0100-29452007000300035.

CAMACHO, M.A.; SILVEIRA, M.V. da; CAMARGO, R.A.; NATALE, W. Faixas normais de nutrientes pelos métodos ChM, DRIS e CND e nível crítico pelo método de distribuição normal reduzida para laranjeira-pera. Revista Brasileira de Ciência do Solo, v.36, p.193-200, 2012. DOI: 10.1590/ S0100-06832012000100020.

CARMO, C.A.F. de S. do; ARAÚJO, W.S. de; BERNARDI, A.C. de C.; SALDANHA, M.F.C. Métodos de análise de tecidos vegetais utilizados pela Embrapa Solos. Rio de Janeiro: Embrapa Solos, 2000. 41p.

CRESTE, J.E.; NAKAGAWA, J. Estabelecimento do método DRIS para a cultura do limoeiro em função da análise foliar. I Cálculo das normas. Revista Brasileira de Fruticultura, v.19, p.295-305, 1997.

DIAS, J.R.M.; PÉREZ, D.V.; SILVA, L.M. da; LEMOS, C. de O.; WADT, P.G.S. Normas DRIS para cupuaçuzeiro cultivado em monocultivo e em sistemas agroflorestais. Pesquisa Agropecuária Brasileira, v.45, p.64-71, 2010. DOI: 10.1590/ S0100-204X2010000100009.

DIAS, J.R.M.; WADT, P.G.S.; PEREZ, D.V.; SILVA, L.M. da; LEMOS, C. de O. DRIS formulas for evaluation of nutritional status of cupuaçu trees. Revista Brasileira de Ciência do Solo, v.35, p.2083-2091, 2011. DOI: 10.1590/S0100-06832011000600023.

DIAS, J.R.M.; WADT, P.G.S.; TUCCI, C.A.F.; SANTOS, J.Z.L.; SILVA, S.V. da. Normas DRIS multivariadas para avaliação do estado nutricional de laranjeira 'Pera' no Estado do Amazonas. Revista Ciência Agronômica, v.44, p.251-259, 2013. DOI: 10.1590/S1806-66902013000200006.

DONAGEMA, G.K.; CAMPOS, D.V.B. de; CALDERANO, S.B.; TEIXEIRA, W.G.; VIANA, J.H.M. (Org.). Manual de métodos de análise de solos. 2.ed. rev. Rio de Janeiro: Embrapa Solos, 2011. 230p. (Embrapa Solos. Documentos, 132).

FAGERIA, N.K.; BARBOSA FILHO, M.P.; MOREIRA, A.; GUIMARÃES, C.M. Foliar fertilization of crop plants. Journal of Plant Nutrition, v.32, p.1044-1064, 2009. DOI: $10.1080 / 01904160902872826$.

FERNANDES, A.R.; REIS, I.N.R.S.; NORONHA, N.C. Estado nutricional de pomares de laranjeira submetidos a diferentes manejos do solo. Revista de Ciências Agrárias, v.53, p.52-58, 2010. DOI: $10.4322 /$ rca.2011.007. 
INSTITUTO BRASILEIRO DE GEOGRAFIA E ESTATÍSTICA. Produção agrícola estadual: lavoura permanente 2011. Disponível em: <http://www.ibge.gov.br/estadosat>. Acesso em: 27 set. 2012.

JARREL, W.M.; BEVERLY, R.B. The dilution effect in plant nutrition studies. Advances in Agronomy, v.34, p.197-224, 1981. DOI: 10.1016/S0065-2113(08)60887-1.

MATTOS JUNIOR, D.; QUAGGIO, J.A.; CANTARELLA, H.; ALVA, A.K. Nutrient content of biomass components of Hamlin sweet orange trees. Scientia Agricola, v.60, p.155-160, 2003. DOI: 10.1590/S0103-90162003000100023.

MCCRAY, J.M.; JI, S.; POWELL, G.; MONTES, G.; PERDOMO, R. Sugarcane response to DRIS-based fertilizer supplements in Florida. Journal of Agronomy and Crop Science, v.196, p.66-75, 2010. DOI: 10.1111/j.1439-037X.2009.00395.x.

MOURÃO FILHO, F. de A.A. DRIS: concepts and applications on nutritional diagnosis in fruit crops. Scientia Agricola, v.61, p.550-560, 2004. DOI: 10.1590/S0103-90162004000500015.

MOURÃO FILHO, F. de A.A.; AZEVEDO, J.C. DRIS norms for 'Valencia' sweet orange on three rootstocks. Pesquisa Agropecuária Brasileira, v.38, p.85-93, 2003. DOI: 10.1590/ S0100-204X2003000100012.

MOURÃO FILHO, F. de A.A.; AZEVEDO, J.C.; NICK, F.A. Funções e ordem da razão dos nutrientes no estabelecimento de normas DRIS em laranjeira 'Valência'. Pesquisa Agropecuária Brasileira, v.37, p.185-192, 2002. DOI: 10.1590/ S0100-204X2002000200010.

PARENT, L.-E. Diagnosis of the nutrient compositional space of fruit crops. Revista Brasileira de Fruticultura, v.33, p.321-334, 2011. DOI: 10.1590/S0100-29452011000100041.

PARTELLI, F.L.; VIEIRA, H.D.; CARVALHO, V.B. de; MOURÃO FILHO, F. deA.A. Diagnosis and recommendation integrated system norms, sufficiency range, and nutritional evaluation of Arabian coffee in two sampling periods. Journal of Plant Nutrition, v.30, p.1651-1667, 2007. DOI: 10.1080/01904160701615525.

PARTELLI, F.L.; VIEIRA, H.D.; MONNERAT, P.H.; VIANA, A.P. Estabelecimento de normas DRIS em cafeeiro conilon orgânico ou convencional no Estado do Espírito Santo. Revista Brasileira de Ciência do Solo, v.30, p.443-451, 2006. DOI: 10.1590/S0100-06832006000300006.

POLITI, L.S.; FLORES, R.A.; SILVA, J.A.S. da; WADT, P.G.S.; PINTO, P.A. da C.; PRADO, R. de M. Estado nutricional de mangueiras determinado pelos métodos DRIS e CND. Revista Brasileira de Engenharia Agrícola e Ambiental, v.17, p.11-18, 2013. DOI: $10.1590 /$ S1415-43662013000100002.
QUAGGIO, J.A.; MATTOS JUNIOR, D.; CANTARELLA, H. Manejo da fertilidade do solo na citricultura. In: MATTOS JUNIOR, D. de; DE NEGRI, J.D.; PIO, R.M.; POMPEU JUNIOR, J. (Ed.). Citrus. Campinas: Instituto Agronômico de Campinas, 2005. p.483-507.

SANTANA, J. das G.; LEANDRO, W.M.; NAVES, R.V.; CUNHA, P.P. da. Normas DRIS para interpretação de análises de folha e solo, em laranjeira Pêra, na região central de Goiás. Pesquisa Agropecuária Tropical, v.38, p.109-117, 2008.

SANTANA, J. das G.; LEANDRO, W.M.; NAVES, R.V.; CUNHA, P.P. da; ROCHA, A.C. Estado nutricional da laranja Pêra na região central do Estado de Goiás avaliada pelas análises foliar e do solo. Bioscience Journal, v.23, p.40-49, 2007.

SILVA, G.G.C. da; NEVES, J.C.L.; ALVAREZ V., V.H.; LEITE, F.P. Avaliação da universalidade das normas DRIS, M-DRIS e CND. Revista Brasileira de Ciência do Solo, v.29, p.755-761, 2005. DOI: 10.1590/S0100-06832005000500011.

VASCONCELOS, L.F.L.; RIBEIRO, R.V.; OLIVEIRA, R.F.; MACHADO, E.C. Variação da densidade de fluxo de seiva e do potencial hídrico foliar nas faces leste e oeste da copa de laranjeira 'Valência'. Revista Brasileira de Fruticultura, v.32, p.35-46, 2010. DOI: 10.1590/S0100-29452010005000004.

WADT, P.G.S. Relationships between soil class and nutritional status of coffee crops. Revista Brasileira de Ciência do Solo, v.29, p.227-234, 2005. DOI: 10.1590/ S0100-06832005000200008.

WADT, P.G.S.; ANGHINONI, I.; GUINDANI, R.H.P.; LIMA, A.S.T. de; PUGA, A.P.; SILVA, G.S. da; PRADO, R. de M. Padrões nutricionais para lavouras arrozeiras irrigadas por inundação pelos métodos da CND e chance matemática. Revista Brasileira de Ciência do Solo, v.37, p.145-156, 2013. DOI: 10.1590/ S0100-06832013000100015.

WADT, P.G.S.; DIAS, J.R.M. Normas DRIS regionais e inter-regionais na avaliação nutricional de café conilon. Pesquisa Agropecuária Brasileira, v.47, p.822-830, 2012. DOI: 10.1590/ S0100-204X2012000600013.

WADT, P.G.S.; SILVA, D.J. Acurácia do diagnóstico nutricional de pomares de mangueiras obtido por três fórmulas DRIS. Pesquisa Agropecuária Brasileira, v.45, p.1180-1188, 2010. DOI: 10.1590/ S0100-204X2010001000018.

WALWORTH, J.L.; WOODARD, H.J.; SUMNER, M.E. Generation of corn tissue norms from a small, high-yield data-base. Communications in Soil Science and Plant Analysis, v.19, p.563-577, 1988. DOI: 10.1080/00103628809367959.

Recebido em 13 de fevereiro de 2013 e aprovado em 28 de junho de 2013 